\title{
Morphometric analysis of the arteries of Willis Polygon
}




\title{
Morphometric analysis of the arteries of Willis Polygon
}

\author{
Huseyin Canaz ${ }^{1}$, Murat Arslan ${ }^{2}$, Husniye Hacioglu ${ }^{3}$, Mehmet \\ Tokmak $^{4}$, Gokhan Canaz ${ }^{5}$, Safiye Cavdar ${ }^{6}$
}

${ }^{1}$ Florence Nightingale Hospital, Istanbul Bilim University, Department of Neurosurgery, Istanbul, TURKEY

${ }_{2}^{2}$ Istanbul Forensic Science Institute, Morgue Department, Istanbul, TURKEY

${ }^{3}$ Marmara University School of Medicine, Department of Anatomy, Istanbul, TURKEY

${ }^{4}$ Istanbul Medipol University, Department of Neurosurgery, Istanbul, TURKEY

${ }^{5}$ Bakirkoy Research and Training Hospital for Neurology, Neurosurgery and Psychiatry, Department of Neurosurgery, Istanbul, TURKEY

${ }^{6}$ Koc University School of Medicine, Department of Anatomy, Istanbul, TURKEY

\begin{abstract}
Objective: Willis polygon forms the basis of the arterial circulation of the cerebrum. Willis polygon is a vascular structure whom variations are not rare. Knowledge of the anatomy and preservation of its integrity is crucial for performing neurovascular surgery and intracranial tumour surgery. Because of the important vascular and neurological structures, approaches to this region are considered extremely risky. One of the main variations in-person basis is the diameter differences of the arteries, which forms Willis polygon, between the left and right hemispheres. About structure and variations, studies of Rhoton and Yasargil had formed the touchstone. Our aim is to contribute to the literature and clinical studies, to be done in the future, by comparing our results with previous studies about variations and morphometric features of Willis polygon. Methods: Arteries of 30 fresh cadaver brains were examined during autopsies in T.C. Ministry of Justice Istanbul Forensic Science Institute. Bilaterally anterior cerebral artery A1 segment lengths, distance between anterior communicating artery-callosomarginal artery outputs, posterior cerebral artery P1 segment lengths were measured using a digital calliper. After dissections and measures, photos of the region were taken and vascular anatomy and variations noted. From every single cerebrum samples were obtained from bilaterally A1, A2, callosomarginal artery, middle cerebral artery, posterior communicant artery, P1 and basilar artery. Samples were fixed by using $10 \%$ buffered-formalin. Taken samples were transported to Marmara University Faculty of Medicine, Department of Anatomy Laboratory. Samples were examined and interior diameters were measured under the microscope. Results: Our results with artery diameters and lengths were similar with literature. Different from literature, in anterior cerebral artery A1 segment, posterior cerebral artery P1 segment and posterior
\end{abstract}


communicant artery no aplasia were noted. In $50 \%$ of the samples, callosomarginal artery were originate from A2 segment. In one case, we observed left and right pericallosal arteries were joined together at the end of the A2 segment and continued as a single pericallosal artery. We could not find any information about this variation in the literature. Conclusion: Before surgical operations, detailed knowledge of Willis polygon and evaluation of the pre-op cerebral angiography considering possible variations, reduce mortality and morbidity ratios. In addition, because of the role of flow gradients of Willis polygon in aneurysm formation, and in terms of better understanding the collateral circulation which is important in vascular occlusive diseases and vascular surgery, we believe, more anatomic studies about this region needed.

Key words: Willis polygon, Circle of Willis, morphometric analysis

\section{Introduction}

Willis polygon forms the basis of the arterial circulation of the cerebrum (Figure 1). Willis polygon is a vascular structure whom variations are not rare $(1,3)$. Knowledge of the anatomy and preservation of its integrity is crucial for performing neurovascular surgery and intracranial tumour surgery. Because of the important vascular and neurological structures, approaches to this region are considered extremely risky (16). One of the main variations in-person basis is the diameter differences of the arteries, which forms Willis polygon, between the left and right hemispheres (5). About structures and variations, studies of Rhoton and Yasargil had formed the touchstone $(10,16)$.

Our aim is to contribute to the literature and to the clinical studies, to be done in the future, by comparing our results with previous studies about variations and morphometric features of Willis polygon.

\section{Material and method}

To evaluate the morphological characteristics of the Willis polygon and its constituent arteries in the Turkish population, arteries of 30 fresh cadaver brains (60 hemispheres) were examined during routine autopsies in T.C. Ministry of Justice Istanbul Forensic Science Institute. All the protocols were conducted with the consent of T.C. Ministry of Justice Istanbul Forensic Science Institute, Board of Science. For the study, cadavers aged between 25 - 50 years old, without head injury were selected. During routine autopsies, after calvarium removal, cerebrums were removed with caution by preserving vessels. On removed cerebrums, arteries of Willis polygon were dissected macroscopically and examined (Figure 2). Bilaterally anterior cerebral artery (ACA) A1 segment lengths, distance between anterior communicating artery (AComA)callosomarginal artery (CMA) outputs, posterior communicating artery (PComA), posterior cerebral artery(PCA) P1 segment lengths were measured using digital calliper. After dissections and measurements, vascular anatomy and variations were documented by taking photos of the region.

From every single cerebrum, samples were obtained from bilaterally A1, A2, CMA, middle cerebral artery (MCA), PComA, P1 and basilar artery (BA). Samples were fixed by using $10 \%$ 
buffered-formaldehyde. Taken samples were examined and interior diameters were measured under a microscope in Marmara University Faculty of Medicine, Department of Anatomy Laboratory (Figure 3).

Statistical evaluations were made using The Statistical Package for Social Scienses (SPSS) 22.0 (Chicago, IL). All continuous variables were reported as median \pm standart deviations (SD).

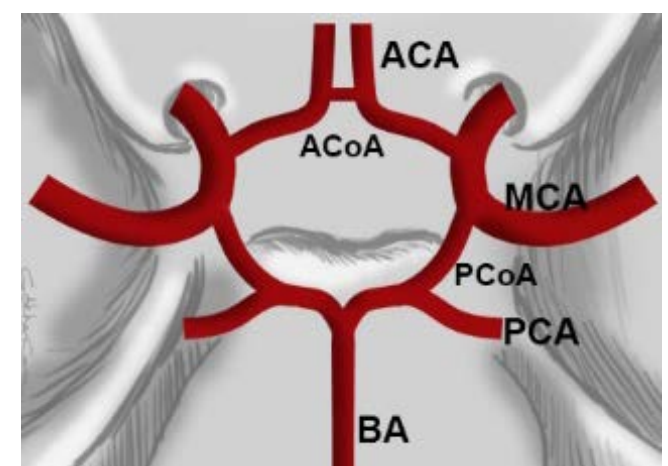

Figure 1 - Illustration of arteries that form Willis Polygon. ACA: Anterior cerebral artery, ACoA: Anterior communicating artery, MCA: Middle cerebral artery, PCoA: Posterior communicating artery, PCA: Posterior cerebral artery, BA: Basilar artery

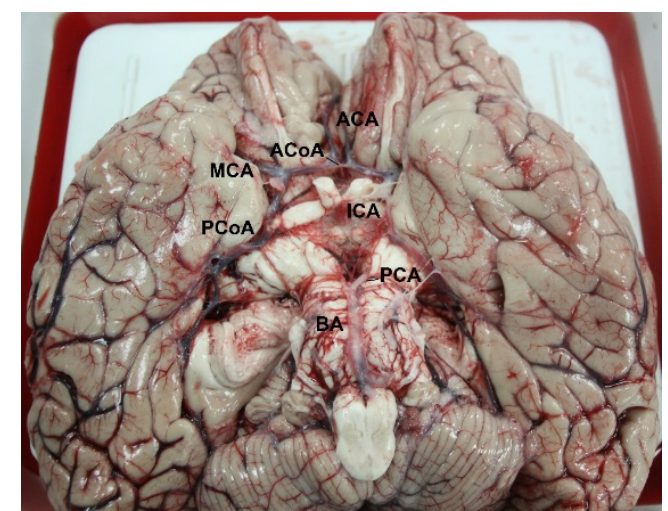

Figure 2 - Arteries of Willis Polygon. ACA: Anterior cerebral artery, ACoA: Anterior communicating artery, MCA: Middle cerebral artery, PCoA: Posterior communicating artery, PCA: Posterior cerebral artery, BA: Basilar artery

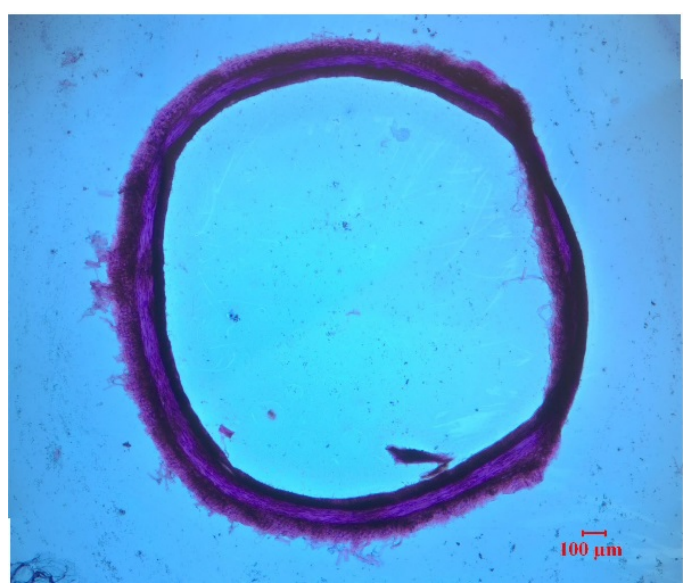

Figure 3 - Microscopic image of an artery sample

\section{Results}

In our study, we used 30 cadaver cerebrums with no head trauma (14 female, 16 male).

\section{A1 Segment}

A1 segment diameters on the right were measured as maximum $2104.593 \mu$, minium $1039.152 \mu$ and median was $1540.092 \pm 366.9 \mu$. On the left, maximum $2699.584 \mu$, minimum $1247.152 \mu$ and median was $1841.398 \pm 355.0 \mu$ (table 1).

A1 segment lengths on the right were measured as maximum $18 \mathrm{~mm}$, minimum $10 \mathrm{~mm}$ and median was $13.56 \pm 2.25 \mathrm{~mm}$. On the left, maximum $17 \mathrm{~mm}$, minimum $10 \mathrm{~mm}$ and median was $13.76 \pm 1.87 \mathrm{~mm}$ (table 1) (Figure 4).

\section{A2 Segment}

A2 segment diameters on the right were measured as maximum $2185.284 \mu$, minimum $1627.145 \mu$ and median was $1860.174 \pm 129.5 \mu$. On the left, maximum $2148.649 \mu$, minimum $1699.004 \mu$ and median was $1841.061 \pm 14.6 \mu$ (table 1).

A2 segment lengths on both right and left were measured as maximum $25 \mathrm{~mm}$, 
minimum $14 \mathrm{~mm}$. Median on the right was $18.83 \pm 3.81 \mathrm{~mm}$ and on the left was $18.73 \pm 3.02 \mathrm{~mm}$ (table 1) (Figure 4).

\section{Posterior Communicating Artery}

PComA diameters on the right were measured maximum $1394.94 \mu$, minimum $638.17 \mu$ and median was $881.377 \pm 170.9 \mu$. On the left, maximum $1410.156 \mu$, minimum $648.95 \mu$ and median was $892.247 \pm 201.4 \mu$.

Posterior communicant artery lengths on the right were measured as maximum $26 \mathrm{~mm}$, minimum $11 \mathrm{~mm}$ and median was $15.63 \pm 3.39 \mathrm{~mm}$. On the left, maximum $24 \mathrm{~mm}$, minimum $11 \mathrm{~mm}$ and median was $15.9 \pm 3.24 \mathrm{~mm}$ (table 1 ).

\section{P1 Segment}

P1 segment diameters on the right were measured as maximum $2529.639 \mu$, minimum $902.703 \mu$ and median was $1807.549 \pm 402.42 \mu$. On the left, maximum $2548.47 \mu$, minimum $948.29 \mu$ and median was $1809.082 \pm 350.05 \mu$ (table 1).

P1 segment lengths on the right were measured as maximum $11 \mathrm{~mm}$, minimum $5 \mathrm{~mm}$ and median was $7.033 \pm 1.44 \mathrm{~mm}$. On the left, maximum $10 \mathrm{~mm}$, minimum $5 \mathrm{~mm}$ and median was 6.933 $\pm 1.2 \mathrm{~mm}$ (table 1) (Figure 5).

\section{Middle Cerebral Artery}

MCA diameters on the right were measured maximum $3017.994 \mu$, minimum $2645.399 \mu$ and median was $2819.351 \pm 101.01 \mu$. On the left, maximum $2992.678 \mu$, minimum $2649.495 \mu$ and median was $2819.284 \pm 94.18 \mu$.

\section{Basilar Artery}

Basilar artery diameters were maximum $3040.072 \mu$, minimum $1939.17 \mu$ and median was $2722.276 \pm 247.57 \mu$ (Figure 5).

\section{Callosomarginal Artery}

CMA diameters on the right were measured as maximum $1489.14 \mu$, minium $836.447 \mu$ and median was $1232,402 \pm 152,51 \mu$. On the left, maximum $2805.314 \mu$, minimum $932.184 \mu$ and median was $1267,75 \pm 361,99 \mu$ (Figure 6).

CMA was observed in 3 cases only on the right side and in 1 case, only on the left side. In two case bilaterally CMA was absent.

The origins of CMA were, left A3 in 10 cases, left $\mathrm{A} 2$ in 15 cases and right $\mathrm{A} 3$ in 12 cases, right $\mathrm{A} 2$ in 15 cases. In 11 cases, CMA were originated from different segments.

TABLE 1

Diameter and length measurements of arteries of the Willis Polygon as mean, minimum and maximum

\begin{tabular}{lccc}
\hline Blood vessels & Mean & Min. & Max. \\
\hline A1 & & & \\
Diameter $(\boldsymbol{\mu})$ - Right & $1540.092 \pm 366.9$ & 1039.153 & 2104.593 \\
Diameter $(\boldsymbol{\mu})$ - Left & $1841.398 \pm 355.0$ & 1247.152 & 2699.584 \\
Length $(\mathbf{m m})$ - Right & $13.56 \pm 2.25$ & 10 & 18 \\
Length $(\mathbf{m m})$ - Left & $13.76 \pm 1.87$ & 10 & 17 \\
\hline
\end{tabular}


60 | Canaz et al - Morphometric analysis of the arteries of Willis Polygon

\begin{tabular}{|c|c|c|c|}
\hline \multicolumn{4}{|l|}{ A2 } \\
\hline Diameter $(\boldsymbol{\mu})$ - Right & $1860.174 \pm 129.5$ & 1627.145 & 2185.284 \\
\hline Diameter $(\boldsymbol{\mu})$ - Left & $1841.061 \pm 14.6$ & 1699.004 & 2148.649 \\
\hline Length (mm) - Right & $18.83 \pm 3.18$ & 14 & 25 \\
\hline Length (mm) - Left & $18.73 \pm 3.02$ & 14 & 25 \\
\hline \multicolumn{4}{|l|}{ PComA } \\
\hline Diameter $(\boldsymbol{\mu})$ - Right & $881.377 \pm 170.9$ & 638.17 & 1394.94 \\
\hline Diameter $(\boldsymbol{\mu})$ - Left & $892.247 \pm 201.4$ & 648.95 & 1410.156 \\
\hline Length (mm) - Right & $15.63 \pm 3.39$ & 11 & 26 \\
\hline Length (mm) - Left & $15.9 \pm 3.24$ & 11 & 24 \\
\hline \multicolumn{4}{|l|}{ P1 } \\
\hline Diameter $(\boldsymbol{\mu})$ - Right & $1807.549 \pm 402.42$ & 902.703 & 2529.639 \\
\hline Diameter $(\boldsymbol{\mu})$ - Left & $1809.082 \pm 350.05$ & 948.29 & 2548.47 \\
\hline Length (mm) - Right & $7.033 \pm 1.44$ & 5 & 11 \\
\hline Length (mm) - Left & $6.933 \pm 1.2$ & 5 & 10 \\
\hline \multicolumn{4}{|l|}{ MCA } \\
\hline Diameter $(\boldsymbol{\mu})$ - Right & $2819.351 \pm 101.01$ & 2645.399 & 3017.994 \\
\hline Diameter $(\boldsymbol{\mu})$ - Left & $2819.284 \pm 94.18$ & 2649.495 & 2992.678 \\
\hline \multicolumn{4}{|l|}{ BA } \\
\hline Diameter $(\boldsymbol{\mu})$ & $2722.276 \pm 247.57$ & 1939.17 & 3040.072 \\
\hline \multicolumn{4}{|l|}{ CMA } \\
\hline Diameter $(\boldsymbol{\mu})$ - Right & $1232,402 \pm 152,51$ & 836.447 & 1489.14 \\
\hline Diameter $(\boldsymbol{\mu})$ - Left & $1267,75 \pm 361,99$ & 932.184 & 2805.314 \\
\hline
\end{tabular}

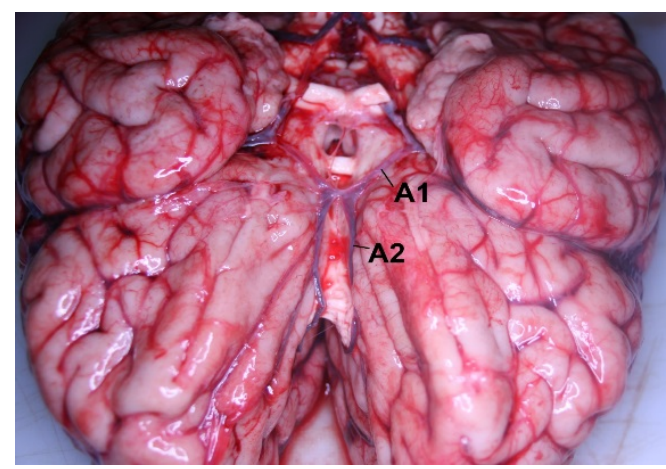

Figure 4 - A1 and A2 segments of ACA

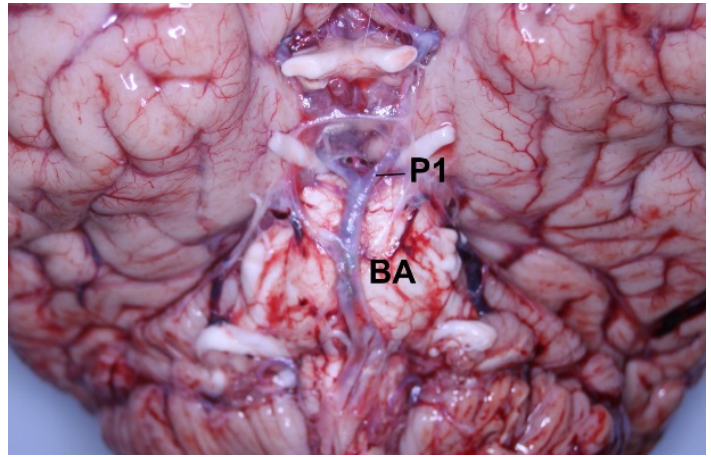

Figure 5 - P1 segment of PCA and BA 


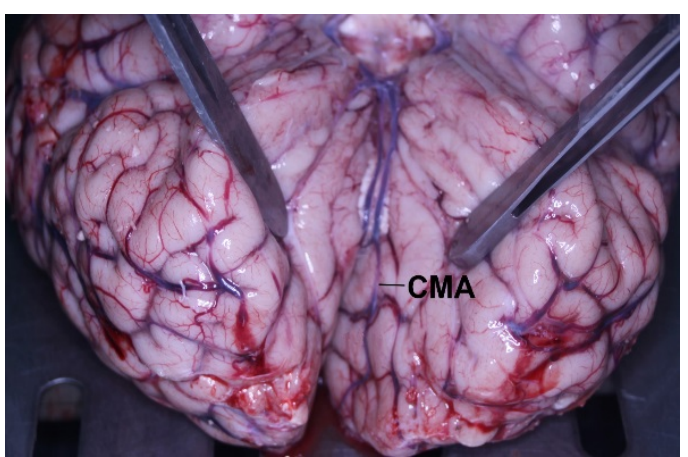

Figure 6 - CMA: Callosomarginal artery

\section{Discussion}

The blood circulation of the cerebrum is formed by 4 arteries as two internal carotid arteries and two vertebral arteries. Internal carotid arteries form the anterior system and vertebral arteries form the posterior system. At the base of the cerebrum, the vascular web that creates the connection between these two systems is named as the "circle of Willis", or "Willis polygon" $(1,10)$

Vessels participating the structure of the polygon are two ICA's, two ACA's, AComA, two PComA's, two PCA's, BA and two MCA's $(1,9)$. Willis polygon provides collateral circulation between vertebrobasilar system and carotid system, and in-between cerebral hemispheres. Direction of flow inside the polygon is determined by vessel diameters and pressure gradient. The efficiency of this collateral circulation is the main determinant of the degree of neurological deficits, in case of stenosis or occlusion in one of the main feeder arteries $(2,6)$.

Many variations in the anatomy of the Willis polygon had been shown and the configuration which could be described as normal is seen in only $50 \%$ of the population
$(6,9,11)$. Approaches to this region are frequently caused by tumours, endovascular interventions and vascular pathologies such as aneurysms and arteriovenous malformations. With better understanding of the region anatomy, lower complication and rates better outcomes would be achieved. Besides differences in configuration, variations in Willis polygon are also caused by differences in vessel diameters and lengths (11).

In our study, in 19 (63\%) cadavers left A1 inner diameters were measured wider and in 11 (37\%) cadavers right A1 inner diameters were wider. Differences were significant in 15 (50\%) cadavers for the left A1 and $8(26.6 \%)$ cadavers for the right A1. In 13 cases (43\%), left $\mathrm{A} 1$ were longer than right $\mathrm{A} 1$ and in 9 cases (30\%) vice versa. In 8 cases (27\%) bilaterally Al's were the same length.

Diameter differences between A1 segments have great value on formation mechanisms of AComA aneurysms. As a general rule, in case of diameter difference between Al segments, AComA aneurysms were originated from the wider side. If the left and right A1 diameters were equal then ACom aneurysms more likely to originate from the middle portion $(14,15)$. It is reported that, in $10 \%, \mathrm{~A} 1$ segment could be one sided hypoplastic or aplastic (12). A1 hypoplasia is associated with anterior communicating artery aneurysms in $85 \%$ of cases. This is the only known variant which is correlated with cerebral aneurysm localization and formation (9). In our study, we did not observe any hypoplastic or aplastic A1 segment. A1 segment aneurysms forms 1.4\% of all intracranial aneurysms (15). Ipsilateral fronto-temporo-sphenoidal craniotomy were 
suggested as most suitable approach for aneurysms of this area (15).

In 19 cadavers $(63 \%)$ right $\mathrm{A} 2$ inner diameter was wider than left A2. In 10 cadavers (33\%) left A2 was wider but the difference was not significant. In one case we observed that after AComA, left ACA was ended with branching on the medial surface of left hemisphere. The right ACA was continuing as the only pericallosal artery, and then in the beginning of $\mathrm{A} 3$ divided and continued as two pericallosal arteries. In literature it is reported that pericallosal arteries might origin from single log, but no ratio was reported (12). In our study, occurance rate of this variation were $3.3 \%$. In addition, in one case we observed that left and right pericallosal arteries were joined together at the end of $\mathrm{A} 2$ and continued as one single pericallosal artery. We could not find any information about this variation in the literature.

In Yasargil's series, pericallosal artery aneurysms are reported as $5.6 \%$ of ACoAACA aneurysms, and as $2.3 \%$ of the all intracranial aneurysms (15). Pericallosal artery aneurysms are mostly seen on A2 segment (4). In our literature research, we noted that on proximal side of CMA and frontopolar artery, aneurysms were more frequent $(1,15)$.

Interhemispheric approach, following paramedian frontal craniotomy, has been been using for pericallosal artery aneurysms (15). Distal ACA aneurysm surgeries have its own difficulties. Because of interhemispheric fissure and callosal cistern are narrow areas, lumbar drainage would be helpful to widen the workspace. Falx depth could be low and consequently there might be adhesions between both cingulate gyruses. Aneurysms of this area are often large based and sclerotic, and frequently involve the vessels which branch from here. Sclerosis could occur on both aneurysm fundus and contralateral pericallosal artery, as a result adhesions between these two structures may occur. This can cause difficulties during dissection. Some of the time, it is hard to tell, from which ACA, the aneurysm was originated and angiography would not be clear. Dome of the aneurysm can be adhered to piamater over cingulate gyrus or it could be buried inside the cingulate gyrus. For all of these, retraction of frontal lobe should be done minimal and with extreme caution. (15).

ACoA is the artery, where the most of the variations observed in Willis polygon (7). It can be as a single line or lines or as a vascular web (10). In our study, in 21 cadavers (70\%) ACoA was as a single line; in 2 cadavers (6.6\%) it was as a conjoint of both ACA's; in 4 cadavers (13.3\%) it was formed by 3 or 4 thin vessels; in 3 cadavers (10\%) it was web-formed. Because of the large number of studies about ACoA and it is a detailed, deep subject; morphological analysis of this artery was not conducted.

In 12 of the cadavers (40\%) left PCA inner diameter was wider than right PCA. In 18 cadavers $(60 \%)$ right PCA was wider. There were significant differences on 7 cases (4 left PCA (13.3\%), 3 right PCA (10\%)). In 18 cadavers $(60 \%)$ we measured the left PCA longer than right, and in 12 cadavers (40) right PCA was longer.

Basilar artery starts with two vertebral arteries joining together in pontomedullary 
sulcus area and continues through prepontine cistern. With age, basilar artery may become more twisted, longer and the bifurcation may be positioned at a higher level (16). 15\% of the saccular aneurysms occur in vertebrobasilar system, and $63 \%$ of them occur on basilar bifurcation. The concomitance of anomalies such as hypoplastic PCA or PCA, with basilar bifurcation aneurysms, is significantly high (8).

In 3 of the cadavers (10\%), only left callosomarginal artery were missing. Right callosomarginal artery was missing in only one cadaver (3,3\%). In 2 cadavers (6,6\%) bilaterally callosomarginal arteries were absent. In Rhoton's series, it is reported that callosomarginal artery were missing in $20 \%$ of the examined hemispheres (8)(66). In our study, among 60 hemispheres, the callosomarginal artery was absent in total 8 hemispheres.

Callosomarginal artery is the largest branch of the pericallosal artery and mostly takes origin from the A3 segment, but it can also take origin from A2 or A4 (9). In our study, callosomarginal artery was taking origin from left A3 in 10 cases, left A2 in 15 cases, right $\mathrm{A} 3$ in 12 cases and right $\mathrm{A} 2$ in 15 cases. In 11 cases, callosomarginal arteries origins were different segments. In total 60 hemispheres, 22(36.6\%) of the callosomarginal artery were originated from A3 segments and 30 of the arteries (50\%) were originated from A2 segments.

We saw no significant relationship between artery lengths and diameters.

In our study, our results were consistent with the literature most of the time. It is reported that $\mathrm{A} 1, \mathrm{P} 1$ and $\mathrm{PCoA}$ segments could be aplastic in 10\%; but we did not encounter this variation in any of our cases.

\section{Conclusion}

Flow strength, intravascular pressure, turbulent flow and artery wall disorders in bifurcation areas takes part in aneurysm pathophysiology (13). Willis polygon, which provides a collateral circulation between carotid and vertebrobasilar system, is a place that intracerebral aneurysms frequently take place. The gold standard treatment of the intracranial aneurysm is still the clipping. Mastering the anatomy of Willis polygon and pre-operative analyses of angiography, considering possible variations, is crucial to decrease mortality and morbidity of this serious operations. In terms of better understanding the collateral circulation which is important in vascular occlusive diseases and vascular surgery, we believe, more anatomic studies about this region needed.

\section{Correspondence}

Gokhan Canaz, Bakirkoy Research and Training

Hospital for Neurology, Neurosurgery and

Psychiatry, Department of Neurosurgery 34147

Bakırkoy/Istanbul. Tel: +90 2124091515 Mobile:

+905369449439

Email: gokhancanaz@gmail.com

\section{References}

1Alpers BJ, Berry RG, Paddison RM. Anatomical studies of the circle of Willis in normal brain. AMA archives of neurology and psychiatry 1959;81:409-418.

2Arnold U, Lehmann R, Kursawe H-K, Lückel W. Hypoplasia of vertebrobasilar arteries; in du Boulay G, Molyneux A, Moseley I (eds): Proceedings of the XIV 
Symposium Neuroradiologicum: London, 17-23 June 1990. Berlin, Heidelberg, Springer Berlin Heidelberg, 1991, pp 426-427.

3Bouthillier A, van Loveren HR, Keller JT. Segments of the internal carotid artery: a new classification. Neurosurgery 1996;38:425-432; discussion 432-423.

4Cinnamon J, Zito J, Chalif DJ, Gorey MT, Black KS, Scuderi DM, Hyman RA. Aneurysm of the azygos pericallosal artery: diagnosis by MR imaging and MR angiography. AJNR American journal of neuroradiology 1992;13:280-282.

5Morris P. Practical Neuroangiography. Lippincott Williams \& Wilkins, 2007.

6Mumenthaler M, Toole JF, Patel AN, Caffi J, Iff-Knopf K. Zerebro-vaskuläre Störungen: Mit Kapiteln über angewandte Embryologie, Anatomie der Gefäße und Physiologie des Gehirns und des Rückenmarks. Springer Berlin Heidelberg, 2013.

7Nathal E, Yasui N, Sampei T, Suzuki A. Intraoperative anatomical studies in patients with aneurysms of the anterior communicating artery complex. Journal of neurosurgery 1992;76:629-634.

8Rhoton AL, Jr. Aneurysms. Neurosurgery 2002;51:S121158.
9Rhoton AL, Jr. The supratentorial arteries. Neurosurgery 2002;51:S53-120.

10Rhoton AL, Jr., Saeki N, Perlmutter D, Zeal A. Microsurgical anatomy of common aneurysm sites. Clinical neurosurgery 1979;26:248-306.

11 Saeki N, Rhoton AL, Jr. Microsurgical anatomy of the upper basilar artery and the posterior circle of Willis. Journal of neurosurgery 1977;46:563-578.

12Schünke M, Schulte E, Schumacher U. Prometheus Lernatlas der Anatomie: Kopf, Hals und Neuroanatomie ; 115 Tabellen. Thieme, 2009.

13Toole JF, Murros K, Veltkamp R. Cerebrovascular Disorders. Lippincott Williams \& Wilkins, 1999.

14Ujiie H, Liepsch DW, Goetz M, Yamaguchi R, Yonetani H, Takakura K. Hemodynamic study of the anterior communicating artery. Stroke 1996;27:20862093; discussion 2094.

15Yaşargil MG. Microneurosurgery. Georg Thieme Verlag, 1984.

16Yaşargil MG. Microneurosurgery vol.1. Georg Thieme Verlag, 1984 